\title{
In Vivo Evaluation of Transdermal Iodide Microemulsion for Treating Iodine Deficiency Using Sprague Dawley Rats
}

\author{
Alaadin Alayoubi, ${ }^{1}$ Ryan D. Sullivan, ${ }^{3}$ Hao Lou, ${ }^{4}$ Hemlata Patel, ${ }^{5}$ Timothy Mandrell, ${ }^{3}$ \\ Richard Helms, ${ }^{2}$ and Hassan Almoazen ${ }^{1,6}$
}

Received 27 May 2015; accepted 9 August 2015; published online 20 August 2015

\begin{abstract}
The objective of this study was to evaluate the transdermal efficiency of iodide microemulsion in treating iodine deficiency using rats as an animal model. Animals were fed either iodine-deficient diet $(20 \mu \mathrm{g} / \mathrm{kg}$ iodide $)$ or control diet $(200 \mu \mathrm{g} / \mathrm{kg}$ iodide $)$ over a 17 -month period. At month 14, iodide microemulsion was applied topically in iodine-deficient group and physiological evaluations of thyroid gland functions were characterized by monitoring the thyroid hormones $\left(\mathrm{T}_{3}, \mathrm{~T}_{4}\right)$, thyroidstimulating hormone (TSH), iodide ion excretion in urine, and the overall rat body weights in both groups. Moreover, morphological evaluations of thyroid gland before and after treatment were performed by ultrasound imaging and through histological assessment. Prior to microemulsion treatment, the levels of $\mathrm{T}_{3}, \mathrm{~T}_{4}$, and TSH in iodine-deficient group were statistically significant as compared to that in the control group. The levels of $\mathrm{T}_{3}$ and $\mathrm{T}_{4}$ increased while TSH level decreased significantly in iodine-deficient group within the first 4 weeks of treatment. After treatment, iodide concentration in urine increased significantly. There was no statistical difference in weight between the two groups. Ultrasound imaging and histological evaluations showed evidence of hyperplasia in iodine-deficient group. Topical iodide microemulsion has shown a promising potential as a novel delivery system to treat iodine deficiency.
\end{abstract}

KEY WORDS: deficiency; iodide; microemulsion; thyroid; transdermal.

\section{INTRODUCTION}

Iodine is a trace element that plays a vital role in the normal function of the thyroid gland and is a primary constituent of thyroid hormones (1). Notably, $30 \%$ of the total amount of iodine in the body $(30-50 \mathrm{mg}$ ) is present in the thyroid gland and forms its hormones $\left(\mathrm{T}_{3}\right.$, triiodothyronine

${ }^{1}$ Department of Pharmaceutical Sciences, University of Tennessee Health Sciences Center, 881 Madison Ave, Memphis, Tennessee 38163, USA.

${ }^{2}$ Department of Clinical Pharmacy \& Center for Pediatric Pharmacokinetics and Therapeutics, University of Tennessee Health Science Center, 881 Madison Ave, Memphis, Tennessee 38163, USA.

${ }^{3}$ Department of Comparative Medicine, University of Tennessee Health Science Center, 956 Court, Memphis, Tennessee 38163, USA.

${ }^{4}$ UPM Pharmaceuticals, 501 5th Street, Bristol, Tennessee 37620, USA.

${ }^{5}$ School of Pharmacy, University of Mississippi, University, Oxford, Mississippi 38677, USA.

${ }^{6}$ To whom correspondence should be addressed. (e-mail: halmoaze@uthsc.edu)

ABBREVIATIONS: $\mathrm{T}_{3}$, Triiodothyronine; $\mathrm{T}_{4}$, Tetraiodothyroxine; TSH, Thyroid-stimulating hormone; TRH, Thyrotropin-releasing hormone; NIS, Sodium/iodide symporter; HPT, Hypothalamic pituitary thyroid; WHO, World Health Organization; KI, Potassium iodide; ID, Iodine deficient; IACUC, Institutional Animal Care and Use Committee; EDTA, Ethylenediaminetetraacetic acid; C-cells, Parafollicular cells; Tg, Thyroglobulin; TPO, Thyroperoxidase. and $\mathrm{T}_{4}$, tetraiodothyroxine) (2). About $60 \%$ of the thyroid iodine is nonhormonal and is concentrated in the extrathyroidal tissues, where its biological role is still unknown (2). The synthesis of $T_{3}$ and $T_{4}$ originates in the thyroid gland triggered by active transport of iodide ion from bloodstream into the thyrocytes via $\mathrm{Na}^{+} / \mathrm{I}^{-}$symporter (NIS) (3). Once the iodide is translocated into the follicular lumen, it undergoes an oxidation reaction forming iodine, then gets incorporated into tyrosine residues in thyroglobulin by thyroid peroxidase, forming precursors $\mathrm{T}_{3}$ and $\mathrm{T}_{4}$ (4). The final synthesis step is proteolytic cleavage of follicular thyroglobulin to release $T_{3}$ and $\mathrm{T}_{4}$ into the bloodstream (5). Adequate levels of thyroid hormones are needed to sustain and prevent any complications in the endocrine thyroidal system (6-8). Thyroid hormones are ordinarily responsible for regulation of body metabolism and energy homeostasis. They actually work on nearly all tissues in the body as they stimulate the metabolic rate, affect protein synthesis, and support bone growth and neural maturation (9). In fact, thyroid hormones are responsible for the normal development and regulation of brain, neurons, and processes that involve cerebral functions (10). A unique and specific mechanism that regulates thyroid hormones is the hypothalamic-pituitary-thyroid (HPT) axis which, as the name indicates, incorporate the hypothalamus, the pituitary gland, and the thyroid gland into forming a loop mechanism that keeps the concentration of these hormones at normal levels (11). This mechanism is initiated with the detection of low levels of thyroid hormones by the hypothalamus. 
This triggers the release of thyrotropin-releasing hormone (TRH). Consequently, TRH stimulates pituitary gland to secrete thyroid-stimulating hormone (TSH). TSH is a stimulator of the thyroid gland and causes an increase in the production of thyroid hormones $\mathrm{T}_{3}$ and $\mathrm{T}_{4}$ until their blood levels is returned to normal. Once these two hormone levels are back to normal, a negative feedback mechanism is initiated over both the hypothalamus and the anterior pituitary glands to stop or reduce the release of TRH and TSH, respectively (12).

Since iodine is essential for the maintenance of normal levels of thyroid hormones, any deficiency of iodine leads inevitably to disruption of HPT axis which gradually advances to multiple adverse effects on growth and development particularly in populations sensitive to iodine deficiency such as pregnant women and their fetuses (13). Interestingly, it was found that inadequate iodine intake during pregnancy, lactation, or early childhood was linked to central nervous system dysplasia causing a range of intellectual, motor, and hearing deficits which are mostly irreversible (14). In older populations, iodine deficiency impairs thyroid hormone synthesis, resulting in hypothyroidism and causing thyroid enlargement (goiter). It can also cause a compensatory increase in the cosubstrate $\mathrm{H}_{2} \mathrm{O}_{2}$, which in turn increases the generation of reactive oxygen species (15). A recent study by Morand et al., showed that the generation of $\mathrm{H}_{2} \mathrm{O}_{2}$ is significantly inhibited by iodine in vivo and in vitro (16). Moreover, a recent study by Clero et al., indicated that iodine deficiency increases the incidence of thyroid malignancy (17). However, it is important to realize that the severity of these complications is correlated with the degree of iodine deficiency (18). The longer the depletion of iodine is, the more severe are the symptoms. Furthermore, mild deficiency of iodine has been reported to cause a significant loss of learning capability (about 13.5 intelligence quotient points at population level) (8). Normally, the iodine requirements vary according to age, gender, and state of deficiency. According to WHO, $150 \mu \mathrm{g} /$ day is the average daily amount needed for normal function of thyroid gland in adults. This quantity increases to $225-350 \mu \mathrm{g} /$ day in cases of women during pregnancy or during lactation (14). Despite the availability of iodine in typical diets, iodine deficiency is considered a major health problem that affects close to two billion individuals worldwide (19-21). This problem is even more evident in developed countries, for example, the national nutritional survey (NHANES III 1988-1994), stated that $15 \%$ of the US female adult population is considered iodine deficient $(22,23)$. The WHO led many global public health campaigns across many continents to incorporate iodized salt as a source for iodine and as a replacement for table salt (19). However, many reports indicate that in many areas, the iodine requirements are not always met by iodized salt especially in the most susceptible groups (pregnant, lactating women, and children 6-24 months of age) (14). For instance, in 2008, 88 samples of iodized table salt were analyzed for iodine quantities and it was found that less than half of those tested contained sufficient iodine for optimal health (24). Thereafter, WHO and the United Nations Children's Fund recommended a complementary method of iodine supplementation to ensure optimal iodine nutrition. Moreover, obtaining the daily required dose of iodine orally can be challenging for specific populations such as infants or patients who suffer from intestinal absorption disorders, and they have to use nutritional support for long periods of time. Indeed, deficiencies in essential elements have been reported in patients with malabsorption after long periods of total parenteral nutrition (10), as most parenteral nutrition formulations do not contain iodine. Patients who receive parenteral nutrition might be at high risk of iodine deficiency (25). Thus, transdermal delivery of iodine might represent a valuable alternative route to oral or parenteral delivery. Transdermal delivery provides many advantages such as convenience, noninvasiveness, avoidance of first-pass metabolism, and extended dosing (26). Previously, our group has developed and characterized an iodide microemulsion delivery system for the transdermal delivery of iodide (27). In vitro skin permeation study showed that iodide microemulsion permeates human skin (27). In the work presented herein, we have evaluated the in vivo applicability and efficiency of a topical iodide microemulsion for treating iodine deficiency in Sprague Dawley rats.

\section{MATERIALS AND METHODS}

\section{Materials}

Potassium iodides (KI), Span 20, sodium carbonate, sodium bicarbonate, and HPLC grade methanol were purchased from Sigma-Aldrich (MO, USA). Anhydrous ethanol was obtained from Fisher Scientific (Thermo Fisher Scientific Inc., PA, USA). Capryol® 90 was purchased from Gattefosse (Lyon, France). Sodium hydroxide and alcohol reagent were obtained from Fisher Scientific (NJ, USA). Millipore deionized water was used in this study.

\section{The Preparation of Microemulsion}

The procedure for preparing iodide microemulsion (50 $\mathrm{mg} / \mathrm{mL}$ ) was described previously (27). In brief, microemulsion $(10 \mathrm{~mL})$ was prepared by mixing $(3.080 \mathrm{~mL})$ Span 20, (3.080 mL) ethanol, and (1.540 mL) Capryol 90, respectively. DI water $(2.3 \mathrm{~mL})$ was then added (under continuous stirring) until a transparent mixture (optical monophasic) was obtained. Thereafter, $0.5 \mathrm{~g}$ of KI was added to obtain a concentration of $50 \mathrm{mg} / \mathrm{mL}$ and the mixture was sonicated until all KI crystals were completely dissolved. Iodide microemulsion was administered transdermally using a constant volume topical actuator (Aptar Pharma, NY, USA) that delivers specified volume $(140 \mu \mathrm{L})$ with each actuation (140 $\mu \mathrm{L}$ contains $7 \mathrm{mg} \mathrm{KI}$ ) of the microemulsion (Fig. 1a). The particle size of the microemulsion was $4.51 \pm 0.15 \mathrm{~nm}$. It is well know that particle size will impact the penetration efficiency through the stratum corneum. The smaller the particles the easier for the microemulsion to pass through the skin.

\section{Animals and Microemulsion Treatment}

Sprague Dawley 5- to 6-week-old male rats, $(n=10)$ weighing 150-200 g, were used for this study. Rats were obtained from Harlan Laboratories (MI, USA). Upon arrival, all rats were housed in standard static microisolator cages at the University of Tennessee animal facility. All animal experiments were performed under an approved IACUC 

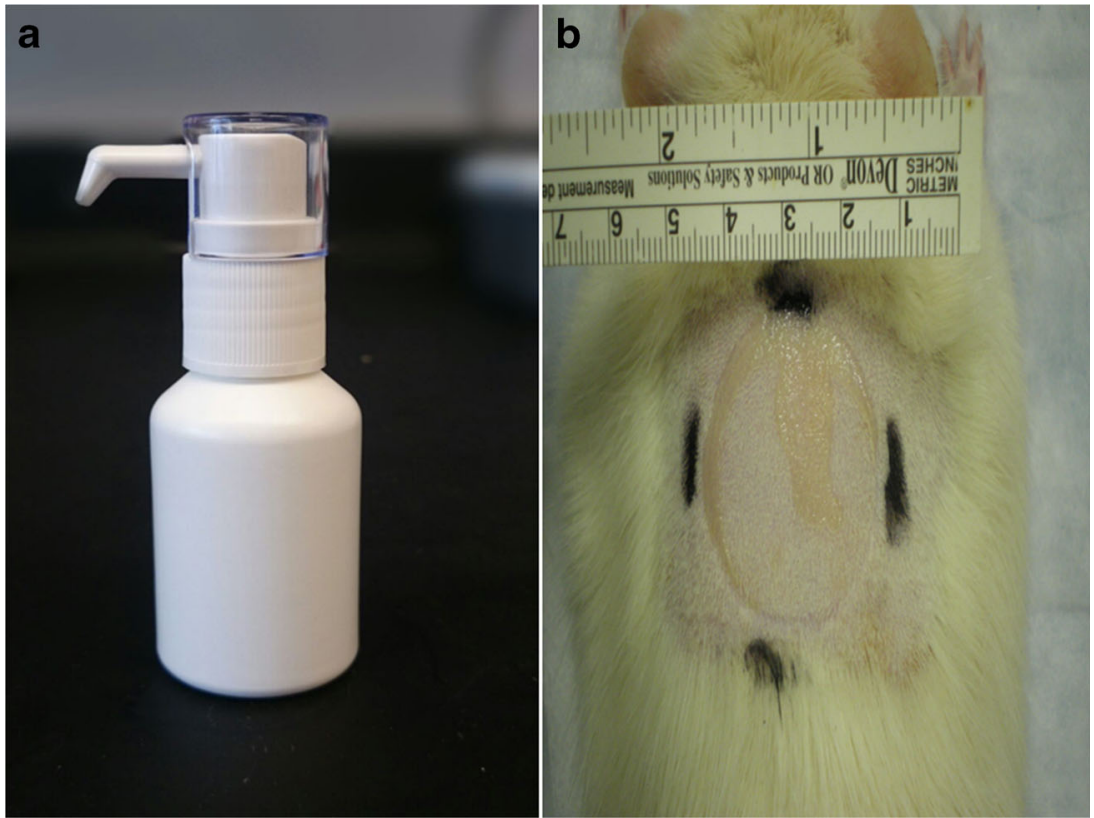

Fig. 1. a Image of a single-dose topical applicator used in delivering iodide microemulsion treatment. This applicator delivers a specified volume $(140 \mu \mathrm{L})$ with each actuation. b Treatment area $\left(7 \pm 0.5 \mathrm{~cm}^{2}\right)$ of iodide microemulsion in iodine-deficient group

(Institutional Animal Care and Use Committee) protocol from the University of Tennessee Health Science Center.

The rats were divided randomly into two groups according to their diet type. The first group was fed iodine-deficient rodent diet $93 \mathrm{G}$ (Harlan Laboratories, MI, USA) that contains $20 \mu \mathrm{g} / \mathrm{kg}$ iodide, and the control group received AIN93G purified diet (Harlan Laboratories, MI, USA) which contains $200 \mu \mathrm{g} / \mathrm{kg}$ iodide. Both diets were provided to the animals throughout the 17 months of the study. The reason for not removing iodine completely in the ID diet is because providing a diet with absolutely no iodine will affect the normal organ functions and the overall growth rate of the rats. In addition, the rats may not survive for the duration of the study. Tap water was tested for iodine content $(<5 \mu \mathrm{g} / \mathrm{L})$ and was freely available. Animals were moved biweekly to metabolism cages in order to collect plasma and urine samples. Rat body weights were recorded using a balance (Tanita KD-160, Arlington Heights, IL, USA) and were collected prior to blood collections while under anesthesia. Blood samples were taken from the tail vein under isoflurane anesthesia. Typically, following microemulsion topical applications, rats were singly housed for 1-2 $\mathrm{h}$ to prevent removal by grooming from other cage mate. Each blood sample $(0.5 \mathrm{~mL})$ was placed in EDTA tubes and centrifuged to separate the plasma. Plasma samples were stored at $-80^{\circ} \mathrm{C}$ for future analysis. Body weights were monitored and recorded biweekly throughout the experiment for both groups. The iodine-deficient group $(n=5)$ was treated weekly after month 14 with $7 \mathrm{mg}$ of KI by applying $140 \mu \mathrm{L}$ of iodide microemulsion transdermally onto a shaved area between the scapulas (shoulder blades) of each rat (Fig. 1b). The treatment surface area was limited to a specified size of $7 \pm$ $0.5 \mathrm{~cm}^{2}$. At the end of the 13 treatments, the animals were sacrificed and thyroid glands were dissected for weighing and histological evaluation.

\section{$\mathbf{T}_{3}, \mathbf{T}_{4}$, and TSH Measurement}

Thyroid-stimulating hormone (TSH) and thyroid hormones $\left(\mathrm{T}_{3}\right.$ and $\left.\mathrm{T}_{4}\right)$ were measured in plasma using rat thyroid hormone immunobead assay MILLIPLEX® MAP kit. The assay procedures were performed as described by the kit's protocol (Millipore, MA, USA). In brief, plasma samples were diluted (1:5) with assay buffer. Thereafter, $25 \mu \mathrm{L}$ of sample was incubated with antibody-coated magnetic beads overnight at $4^{\circ} \mathrm{C}$. Next day, all beads were washed three times using automated magnetic plate washer Tecan HydroFlex ${ }^{\mathrm{TM}}$ (Tecan Group Ltd., Switzerland). After incubation, streptavidinphycoerythrin solution was added per well. Standard calibration curves for known concentrations of $\mathrm{T} 3, \mathrm{~T}_{4}$, and TSH were constructed to convert the fluorescence units into concentration values $(\mathrm{ng} / \mathrm{mL})$. All standards, quality controls, and plasma samples were run in duplicate on BioPlex ${ }^{\circledR}$ (Bio-Rad, Hercules, CA).

\section{Ion Chromatography}

High-pressure ion chromatography (Shimadzu, Japan) equipped with suppressed conductivity detector was used to measure iodide concentration in urine. The instrument consisted of an LC-20AD pump, SIL-20AC HT auto sampler, CTO-20A column oven, CDD-10A VP conductivity detector, and CBM-20A system controller. The system was connected to SeQuant ${ }^{\circledR}$ CARS $^{\text {TM }}$ Suppressor system (Millipore, MA, USA). The suppression system consisted of SeQuant ${ }^{\circledR}$ CARS $^{\mathrm{TM}}$ Suppressor and SeQuant ${ }^{\circledR}$ CARS ${ }^{\mathrm{TM}}$ Cartridge. SeQuant ${ }^{\circledR}$ SAMSTM Suppressor contains an ion exchange membrane and is used for mobile phase background suppression. The SeQuant ${ }^{\circledR}$ CARSTM $^{\text {TM }}$ Cartridge provides continuous autoregeneration for the suppressor column. Anion 
separation was performed on Dionex IonPac AG16/AS16 guard/separation columns (Thermo Scientific, MA, USA). The injection volume was set to $20 \mu \mathrm{L}$. The mobile phase consisted of a mixture of $50 \%$ of [ $1.8 \mathrm{mM}$ sodium carbonate $\left(\mathrm{Na}_{2} \mathrm{CO}_{3}\right)$ and $1.7 \mathrm{mM}$ sodium bicarbonate $\left.\mathrm{NaHCO}_{3}\right]$ and $50 \%$ of [ $5 \mathrm{mM}$ sodium hydroxide] and was used under isocratic conditions at a flow rate of $1 \mathrm{~mL} / \mathrm{min}$. Iodide was detected at retention time of $17 \mathrm{~min}$. The sodium in the mobile phase was efficiently removed by the suppression method which is used to reduce noise and improve sensitivity. The mobile phase was prepared using Millipore ultrapure water and was filtered through a $0.45-\mu \mathrm{m}$ cellulose membrane (Millipore, MA, USA) and then degassed under vacuum.

\section{Urine Purification and Sample Pre-treatment}

The purification process of urine samples was carried out as previously published with modifications (28). In brief, stored urine samples at $-80^{\circ} \mathrm{C}$ were thawed at room temperature. Thereafter, samples were centrifuged at $5000 \mathrm{rpm}$ in accuSpin $^{\text {TM }}$ Micro/Micro R Benchtop Centrifuge (Fisher Scientific Co., NJ, USA) for $10 \mathrm{~min}$ to precipitate any suspended particulate materials and $500 \mu \mathrm{L}$ of the clear supernatant were transferred into a 2-mL eppendorf vial. One and half milliliter of cold alcohol was added to each sample to precipitate proteins. The samples were centrifuged at $5000 \mathrm{rpm}$ in (Thermo Scientific, MA, USA) for 10 min until clear supernatant was acquired. The supernatant was transferred to glass tubes and placed under ultrapure nitrogen stream in $37^{\circ} \mathrm{C}$ water bath until all solvent was evaporated and urinary solid matrix was obtained. The urinary solid material was reconstituted with $500 \mu \mathrm{L}$ of $5 \mathrm{mM} \mathrm{NaOH}$ and vortexed until all solid materials were dissolved. The reconstituted urine samples were passed through $50 \mathrm{mg} / \mathrm{mL}$ Hypersep C18 Extraction Column (Thermo Scientific, MA, USA) for further purification using vacuum. Prior to use, each Hypersep C18 Column was rinsed with 3-mL methanol and then washed with 6-mL ultrapure water. Extra vacuum was applied to remove any residual solvent and to ensure that there was no sample dilution during the pretreatment procedure. Ten samples were purified together using Resprep® 24-Port SPE Manifolds (Restek, PA, USA). The percentage recovery of iodide in sample treatment was determined by passing known concentration of iodide dissolved in DI water $(10 \mu \mathrm{g} / \mathrm{mL})$ through all steps of purification. A recovery of $80 \%$ was calculated and all iodide concentration values were modified accordingly.

\section{High-Frequency Ultrasonography (HFUS) Evaluation}

Vevo 2100 high-resolution micro-imaging system (Visualsonics, Toronto, Ontario, Canada) with a $30-\mathrm{MHz}$ transducer (MS 400; Visualsonics) was used for all ultrasound examinations. Two-dimensional transverse and longitudinal images of both thyroid lobes were obtained using a rail system (Vevo Integrated Rail System 2; Visualsonics). Rats were anesthetized using 1-2\% isoflurane vaporized in oxygen and secured in dorsal recumbencies to the EKG leads on the heated platform stage with a tape. Body temperature, heart rate, and respiratory rate were monitored throughout the procedure to maintain stable physiological parameters. Moreover, the ventral cervical region was removed with depilatory cream (Nair, Church \& Dwight Co. Inc., Princeton, NJ) and a thick layer of coupling gel (Ultrasound Gel, Medline Industries, Inc., Mundelein, IL) was placed over the area of interest. Image analysis was done post-image acquisition using Vevo 1.5 software package. The volume of each lobe was calculated using the ovoid formula (width $\times$ depth $\times$ length $\times \pi / 6$ ) with the linear measurements taken at the widest portion of all scans, excluding the isthmus (29). All ultrasound acquisitions and analysis were performed by the same trained ultrasonographer.

\section{Histological Evaluation}

The rats were euthanized with an overdose of isoflurane and a secondary thoracotomy. The skin was removed over the ventral cervical region and blunt dissection was used to locate the thyroid at the level of the second or third tracheal ring. The complete thyroid, both lobes and isthmus, were excised en bloc using sharp iris scissors. Once removed, the thyroids were individually photographed and weighed using a balance (Adventurer Pro AV264C, Ohaus Corporation, Pine Brook, NJ). The thyroids were placed in $10 \%$ neutral-buffered formalin for $24 \mathrm{~h}$ and then embedded in paraffin blocks following automatic processing. Slides were prepared from $4-\mu \mathrm{m}$ coronal sections stained with hematoxylin and eosin for microscopic analysis. All histological images were analyzed by the same pathologist who was blinded to the group designation and ultrasound results.

\section{Stereology Analysis}

Further evaluation of the histological sections was performed using a similar protocol outlined in Chin wing kot et al., to quantify the colloid differences between groups (30). In summary, 30 individual thyroid follicles from each rat were measured using Image J2 (Fiji/LOCI, Madison, WI) software to calculate average colloid area $\left(\mu \mathrm{m}^{2}\right)$ per follicle. All image measurements were performed at 40.0× magnification. Final results per group are presented as group averages \pm standard deviations.

\section{Statistical Analysis}

Two-tailed Student's $t$ tests were performed on original data to assess statistical differences between the groups. Significance levels were set at $P<0.05$.

\section{RESULTS}

\section{Iodine Deficiency Phase}

The objective of this phase was to induce iodine deficiency in rats and to determine the monthly values of $\mathrm{T}_{3}, \mathrm{~T}_{4}$, and TSH in plasma of the ID and the control groups. Two groups of Sprague Dawley rats (5 to 6 weeks old) were fed with lowiodine diet at $20 \mu \mathrm{g} / \mathrm{kg}$ (iodine deficiency group, ID $n=5$ ) and similar diet with sufficient iodine added at $200 \mu \mathrm{g} / \mathrm{kg}$ (control group $n=5$ ). The plasma levels of $\mathrm{T}_{3}, \mathrm{~T}_{4}$, and TSH in iodinedeficient rats and in the control group were monitored over a 17-month period. Results are depicted in Fig. 2a-c. At the beginning of the study, the thyroid hormones $T_{3}, T_{4}$, and 

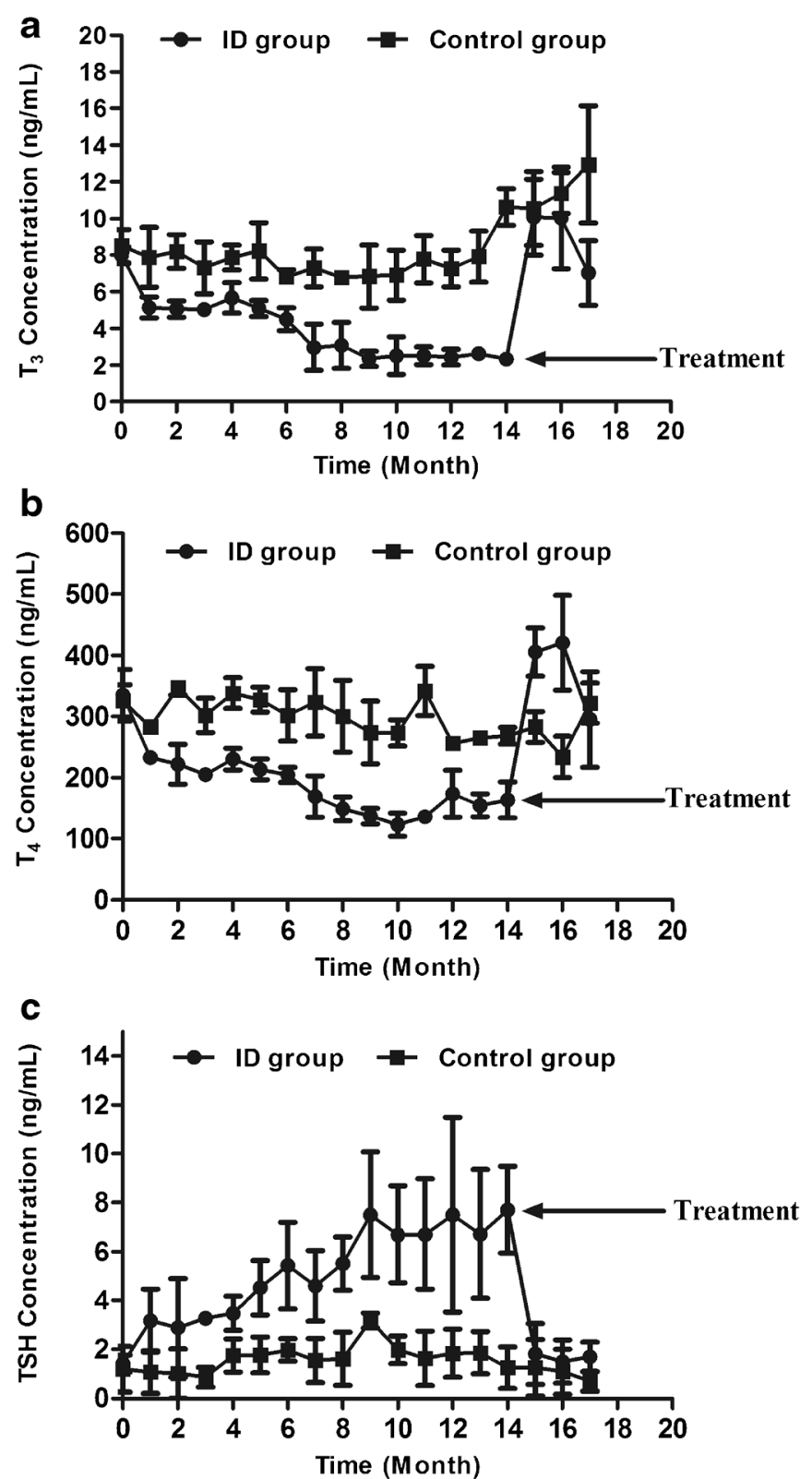

Fig. 2. Plasma concentration (ng/ml) of $\mathbf{a} \mathrm{T}_{3}, \mathbf{b} \mathrm{T}_{4}$, and $\mathbf{c} \mathrm{TSH}$ in iodinedeficient and control groups fed with iodine-sufficient or iodinedeficient diet throughout 17 months of the study ( $n=5$ per group); all data are expressed as means \pm SD

TSH showed similar levels in both groups. At the end of the first month, there was a statistically significant reduction in $T_{3}$ and $\mathrm{T}_{4}$ levels in the ID group as compared to that in the control group $(P<0.001)$. The values of $\mathrm{T}_{3}$ and $\mathrm{T}_{4}$ continued to decrease till it reached the values of $5.2 \pm 0.6$ and $233 \pm 9 \mathrm{ng} /$ $\mathrm{mL}$ in the ID group at month 5 . The values for the control group were bounding around $7.9 \pm 1.6$ and $283 \pm 9 \mathrm{ng} / \mathrm{mL}$ during that period. After month 5, there were further reductions in $T_{3}$ and $T_{4}$ in ID diet until a minimum plateau was reached at month 7 for $T_{3}$ and month 10 for $T_{4}$. The measured values for $\mathrm{T}_{3}$ and $\mathrm{T}_{4}$ at 7 and 10 months were $3 \pm 1.26$ and $123 \pm 20 \mathrm{ng} /$ $\mathrm{mL}$, respectively. These values of $\mathrm{T}_{3}$ and $\mathrm{T}_{4}$ did not change until the introduction of the iodide microemulsion treatment at month 14 (Fig. 2a, b).
$\mathrm{TSH}$, on the other hand, showed an opposite pattern in comparison to $\mathrm{T}_{3}$ and $\mathrm{T}_{4}$. At the beginning of the study (Fig. 2c), the TSH plasma values in ID and control groups were $1.4 \pm 0.4$ and $1.2 \pm 0.9 \mathrm{ng} / \mathrm{mL}$, respectively. During the first and second months, the value of TSH increased in the ID group to $3.2 \pm 1 \mathrm{ng} / \mathrm{mL}$ while maintained at $1.1 \pm 0.9 \mathrm{ng} / \mathrm{mL}$ in the control group. However, this difference of TSH levels was insignificant between the two groups $(P>0.05)$ during these 2 months. During the following several months, the values of TSH in the ID group continued to climb noticeably at months 7 and 8 and to reach maximum TSH value at months 9-12 (average $=7.8 \pm 3 \mathrm{ng} / \mathrm{mL}$ ). Evidently, the increase in $\mathrm{TSH}$ values in these months was significant $(P<0.001)$ in comparison to the control group and remained so until the end of 
14 months of the study. After month 14, the average TSH values remained at $1.5-2 \mathrm{ng} / \mathrm{mL}$ in the control group and remained close to these values during the next 17 months of the study (Fig. 2c).

\section{Transdermal Treatment of the Iodide Microemulsion}

During the iodine deficiency phase (initial 14 months), most of the $T_{3}, T_{4}$, and TSH plasma values in the ID group were statistically significant as compared to that in control groups. Thus, we decided to begin the in vivo evaluation of the iodide microemulsion and measure the hormonal levels pre- and post-treatment. For this reason, a constant dose of microemulsion $(140 \pm 2 \mu \mathrm{L}$ equivalent to $50 \pm 1 \mu \mathrm{g} / \mu \mathrm{L}$ of $\mathrm{KI})$ was provided from a plastic topical applicator and was applied once a week on the shaved craniodorsal thoracic region (shoulder blades) of the rat neck for 3 months. During this time, the control group received no topical treatment. After the beginning of the treatment and during the post-treatment month, a sharp increase in $T_{3}$ and $T_{4}$ values was observed and maintained at similar values during the second month (Fig. 2a, b). $\mathrm{T}_{3}$ levels post-treatment increased about 4-fold from $2.34 \pm 0.2$ to $10 \pm 2 \mathrm{ng} / \mathrm{mL}$ while $\mathrm{T}_{4}$ values increased about 2.25 -fold from $164 \pm 30$ to $405 \pm 40 \mathrm{ng} / \mathrm{mL}$. However, at the end of the third month, a marked reduction in both $\mathrm{T}_{3}$ and $\mathrm{T}_{4}$ was observed in the ID group. The average values of $\mathrm{T}_{3}$ and $\mathrm{T}_{4}$ were $7 \pm 1.8$ and $295 \pm 80 \mathrm{ng} / \mathrm{mL}$, respectively. $\mathrm{T}_{3}$ and $\mathrm{T}_{4}$ posttreatment values in the ID group were higher than average $\mathrm{T}_{3}$ and $\mathrm{T}_{4}$ values in the control group during the first 2 months of treatment. However, at the third month, $\mathrm{T}_{4}$ levels in the ID group became equivalent to $T_{4}$ values in the control group. However, $\mathrm{T}_{3}$ levels in the ID group did not become equivalent to $T_{3}$ levels in the control group. This might be attributed to the unexpected increase of $T_{3}$ levels in the control group at the end of the study.

The post-treatment TSH values exhibited a marked reduction in the average values $(1.6 \pm 1 \mathrm{ng} / \mathrm{mL})$ during the 3 months following the treatment (Fig. 2c). These values were statistically significant $(P<0.0001)$ as compared to TSH values just before treatment $(7.7 \pm 1.8 \mathrm{ng} / \mathrm{mL})$.

\section{Monitoring Urinary Excretion of Iodide}

Since the majority of iodide absorbed into the body is excreted into the urine, the determination of iodide levels in urine becomes a reliable marker for iodide concentration in the plasma. During this study, we detected the iodide secretion in urine in both ID and control groups using 24-h urinary collections and expressing only the end of the month measurements. During this study, the urinary iodide concentrations were expressed in micrograms per milliliter. Details of sample pretreatment and analysis were discussed in "MATERIALS AND METHODS" section. Shortly, after extracting iodide from urine samples, iodide ion was analyzed using highpressure ion chromatography with ion suppression conductivity detector. All data were compiled and presented in Fig. 3. This figure indicates that both ID and control groups exhibited an increase in the amount of iodide ion excreted in urine during the first 2 months of the study. Iodide concentration increased in both groups from about $0.5 \pm 0.5 \mu \mathrm{g} / \mathrm{mL}$ at the beginning of the study to $2 \pm 0.8 \mu \mathrm{g} / \mathrm{mL}$ at month 2 .

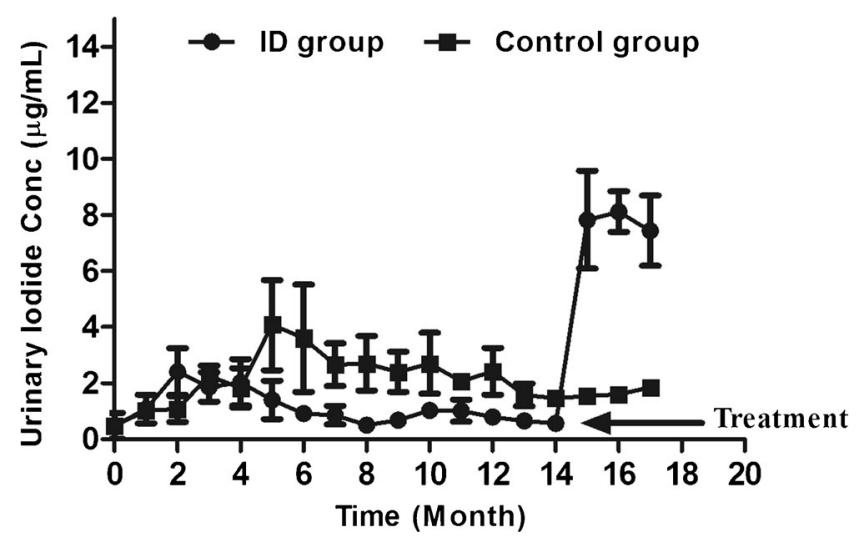

Fig. 3. Iodide concentration $(\mu \mathrm{g} / \mathrm{mL})$ in urine samples taken from rats in iodine-deficient and control groups throughout 17 months of the study ( $n=5$ per group); all data are expressed as means \pm SD

Comparatively, there was no statistical significance in iodide concentrations between the two groups at this early phase of the study $(P>0.05)$. At the end of second month, the iodide urine excretion in the ID group declined while the iodide excretion for the control group continued to rise, and statistically significant differences in iodide levels between the two groups were apparent during months 4 to $14(P<0.0001)$. After month 2, the ID group showed a slight reduction in iodide ion concentration as compared to the control group. The iodide values stayed at this level until it reached a minimum value of $0.5 \pm 0.2 \mu \mathrm{g} / \mathrm{mL}$ at the end of month 14 just before the beginning of the treatment phase. In contrast to the ID group, iodide levels in the control group after month 2 continued to rise until reaching steady values $(4 \pm 2$ and $3.6 \pm$ $2 \mu \mathrm{g} / \mathrm{mL}$ ) during months 5 and 6 , respectively. After month 6 , however, iodide concentration started to decrease progressively down to a value of $1.5 \pm 0.1 \mu \mathrm{g} / \mathrm{mL}$ at the end of month 14. Nevertheless, the differences between the two groups remained significant $(P<0.05)$. Once the microemulsion treatment started at month 14 in the ID group, a sharp increase in iodide levels was observed beginning from 0.5 up to $7.8 \mu \mathrm{g} / \mathrm{mL}$ at month 15 (Fig. 3). The values were stable for the following 2 months post-treatment. This increase was significantly higher as compared to iodide levels at pre-treatment $(P<0.001)$.

\section{Mean Body Weights of Rats}

In order to determine the influence of iodide deficiency on rat growth rate, the mean body weights (BW) of ID and control groups were monitored throughout the study (Fig. 4). Statistical analysis using Paired $t$ test was conducted on the weight data for the ID and control groups. There was no significant difference in the average weight between the two groups throughout the study $(P>0.05)$. However, the growth rate in ID and control groups showed different profiles as the average weight of the ID group needed 7 months to reach $500 \mathrm{~g}$, while the rats in the control group reached the same average weight in 3 months (Fig. 4). Afterwards, the difference continued but remained insignificant between the two groups until the end of the study, indicating no correlation between iodine deficiency and weight. 


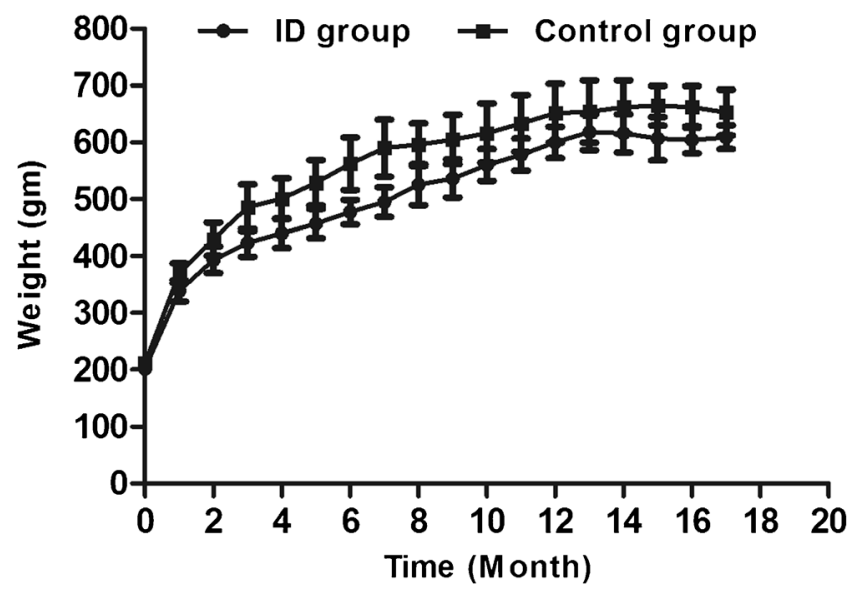

Fig. 4. The average weight of rats in the iodine-deficient and control groups throughout 17 months of the study; all data are expressed as means \pm SD

\section{Ultrasound Imaging}

Ultrasound imaging at the 14-month time point showed a significant difference in thyroid gland sizes of the ID group compared to that in the control group (see the width of right and left lobe in ID rat in Fig. 5a and control rat in Fig. 5b). This marked hyperplasia or goiter of thyroid gland in ID rat was clear at 14 months of evaluation. Following only four topical-microemulsion treatments, the ultrasound evaluation of the ID rats at 15 months revealed a drastic decrease in thyroid gland morphology when compared to that in the control (see the width of thyroid gland of the same rat in Fig. $5 \mathrm{c}$ and the width of thyroid gland of the control rat in Fig. 5d). However, a full return to a euthyroid gland size was still not evident following the final ultrasound evaluation 1 week after the final treatment at month 17 for the ID group (see Fig. 5e, f after 13 topical treatments).

\section{Volume Change of the Thyroid}

Since the rats in the ID and control groups are constantly growing, the size of the thyroid gland is always proportional to the age and the weight of each rat. Thus, we decided to calculate the volume of the thyroid gland per rat from the three-dimensional measurements of the ultrasound imaging assuming an ovoid shape (width $\times$ depth $\times$ length $\times \pi / 6)$ and divide this volume by the weight of each rat. The ratios were averaged to obtain the average ratio per group (Fig. 6a). This figure shows a comparison between the ratios obtained from the ID and control groups. At months 8 and 14 (prior to microemulsion treatment) a statistical significance between the ID group and control was observed $(P<0.05)$. The ratio of the ID group is almost double than that of the control group. This indicates severe thyroid enlargement due to chronic iodine deficiency. At months 15, 16, and 17 (after treatment), the ratios for the ID group was relatively larger in value than the control group and the difference was statistically significant $(P<0.05)$, but this was an indication that the microemulsion treatment is shrinking the volume of the thyroid gland in the ID group. At the end of the experiment, animals were sacrificed and the thyroid glands were removed and weighed per animal from both groups; then the ratio of thyroid gland weight over the weight per rat was plotted to obtain the ratios in Fig. 6b. This figure shows that the average weight ratio for the ID group is almost double than that of the control group $(P<0.05)$ which suggests the thyroid gland has not fully recovered to its normal weight after 3 months of treatment.

\section{Histological Evaluation}

Paired thyroid glands were evaluated for each animal. The ID group had notably larger thyroid glands (goiter) compared to the control group (Fig. 7a, b). Other than size, few differences between groups were noted throughout. All glands had pleomorphic follicles that contained colloid with moderate amounts of basophilic concentrations which was interpreted to be mineral (Fig. 7c, white arrow). Larger amounts of mineral were observed in the ID group (Fig. 7c). The follicles contained numerous pyknotic cells sloughing into the lumens. Parafollicular cells (C-cells) were also sloughing within the follicular lumens, representing a mild parafollicular hyperplasia. A single rat from the control group had bilateral diffuse parathyroid gland hyperplasia. Stereology confirms that there is a significant statistical difference $(P<0.05)$ between the average colloid per follicular cell between the ID $\left(46,096 \pm 20,923 \mu \mathrm{m}^{2}\right)$ and control $\left(106,357 \pm 32,605 \mu \mathrm{m}^{2}\right)$ groups (Fig. 8).

\section{DISCUSSION}

We have developed an iodide microemulsion which has shown promising efficiency in treating iodine deficiency in Sprague Dawley rats (27). The iodide microemulsion was prepared using potassium iodide (KI) as the iodine source. Typically, most iodine formulations are composed of a combination of iodine and sodium iodide (i.e., Lugol's solution, Iodoral). Such formulation contains one-third iodine $(5 \%)$ and two-thirds potassium iodide (10\%) (31). The advantage of administering either iodine or iodide has not been evaluated. However, it has been reported that iodide appears to be more efficient than iodine in restoring the thyroid gland to its normal condition during iodine deficiency (2). In this rat study, we applied the iodide microemulsion on a specific area between the shoulder blades of the rats after trimming the hair at the application site (Fig. 1b), so they would not ingest the microemulsion. This procedure eliminated unequal distribution of the formulation on the skin.

The transdermal treatment was initiated with a single weekly dose of 7-mg KI (equivalent to $5.35 \mathrm{mg}$ of elemental iodide $\mathrm{I}^{-}$) using a topical applicator Aptar ${ }^{\circledR}$ (Fig. 1a). The application of this large dose was implemented for two reasons: (a) to compensate and restore the normal functionality of thyroid gland in a short period of time and (b) to detect any observable physiological toxicity that might arise from the treatment.

The normal values for $\mathrm{T}_{3}, \mathrm{~T}_{4}$, and $\mathrm{TSH}$ vary greatly in the literature. These variations might be attributed to the different analytical methods used to detect the hormones (see Zoeller et al. 2007; Nelson and Wilcox 1996; McLanahan et al. 2008; Liewendahl 1990), composition of the diet, duration of 


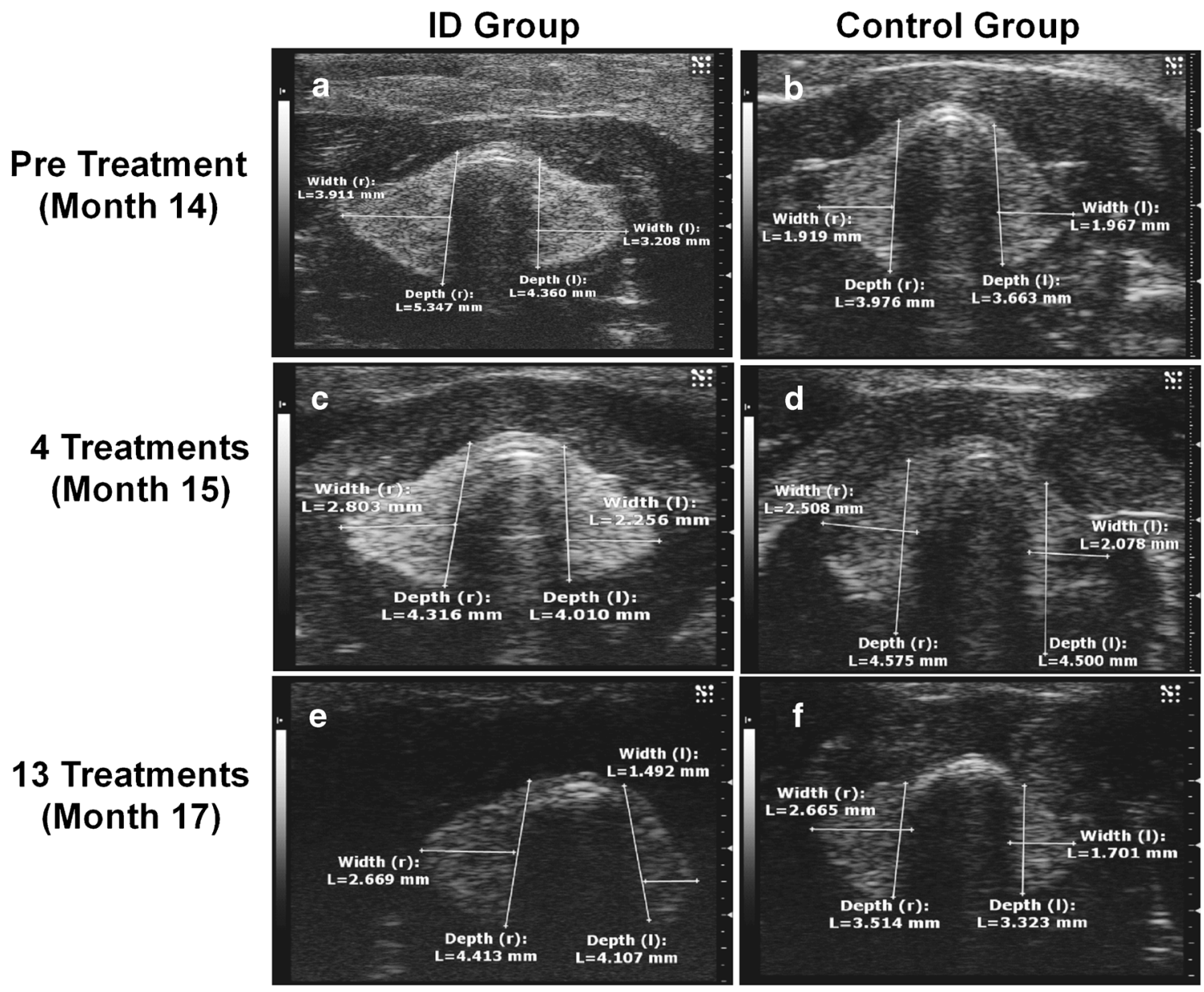

Fig. 5. Ultrasound imaging in transverse views of the thyroid gland at the widest level when scanned from caudal to cranial poles. The image is an evaluation example from a representative animal of the iodine-deficient group (a, $\mathbf{c}, \mathbf{e})$ and from the control group in $(\mathbf{b}, \mathbf{d}, \mathbf{f})$ imaged pre-treatment and post-treatment, respectively. All measurements are in millimeters $(\mathrm{mm})$

experiments, rat age, strain, or gender of the rats (see Heninger and Albright 1975; Versloot et al. 1997; Riesco et al. 1976; Okamura et al. 1981).

Nevertheless, many reports indicated that excess intake of iodine beyond the physiologically required iodine is well tolerated (4). No toxicity was reported in either humans or animals when 3-15 mg/day of iodine were administered (25). In this study, neither changes of behavior nor other toxicity signs were noticed on the ID group. It is well known that the LD 50 of potassium iodide in rats is about $4800 \mathrm{mg} / \mathrm{kg}$. The absence of any harmful effects by excess uptake of iodine is explained by the ability of the thyroid gland to adjust a wide range of intakes. In the short term, this is enforced by the thyroid autoregulation mechanism which is known as the "acute Wolff-Chaikoff effect." Based on this mechanism, when a high amount of iodide (3-10 times the daily required intake) is given to euthyroid subjects, a temporary decrease in the synthesis of thyroid hormones occurs for 24-48 h. This occurs as a result of the high levels of intrathyroid iodine concentrations which inhibits the iodination of the tyrosyl residues on thyroglobulin $(\mathrm{Tg})$ by thyroperoxidase (TPO). Thus, this leads to the reduction in thyroid hormones synthesis. After $48 \mathrm{~h}$, the thyroid gland terminates this effect by adopting alternative mechanism of the so-called the escape phenomenon which decreases the thyroid iodine trap, thus decreasing the intrathyroid iodine concentrations (32). The lapse of toxicity can also be linked to the hydrophilicity of iodide which allows for $90 \%$ of the ingested iodide to be eliminated from the body by urinary excretion. It was reported that only $6-12 \%$ of iodine from iodine solutions can reach blood stream when given through the skin (33). For this reason, applying a significant high dose of iodide is attractive in order to deliver sufficient quantity of iodide which can initiate a hasty return of the thyroid hormones to normal values. When iodide deficiency is prolonged and the typical compensatory mechanism is not able to make up for the loss of iodide, further reduction in $\mathrm{T}_{3}$ and $\mathrm{T}_{4}$ or more production of TSH will eventually lead to goiter and enlargement of the thyroid gland (34).

During the first 14 months of this study, $\mathrm{T}_{3}, \mathrm{~T}_{4}$, and TSH showed a typical iodine deficiency pattern, as $\mathrm{T}_{3}$ and $\mathrm{T}_{4}$ decreased and TSH increased gradually (Fig. 2a-c). The changes in $\mathrm{T}_{3}$ and $\mathrm{T}_{4}$ began during the first month of the experiment. This comes in an agreement with previous reports which showed a significant reduction in $\mathrm{T}_{3}$ and $\mathrm{T}_{4}$ during the first few weeks of the iodine deficiency phase $(35,36)$. Here in our study, we noticed $\mathrm{T}_{3}$ and $\mathrm{T}_{4}$ concentrations started to reduce in the ID group in the first 2 weeks. However, there were previous studies that showed an immediate decrease in $\mathrm{T}_{4}$ but 

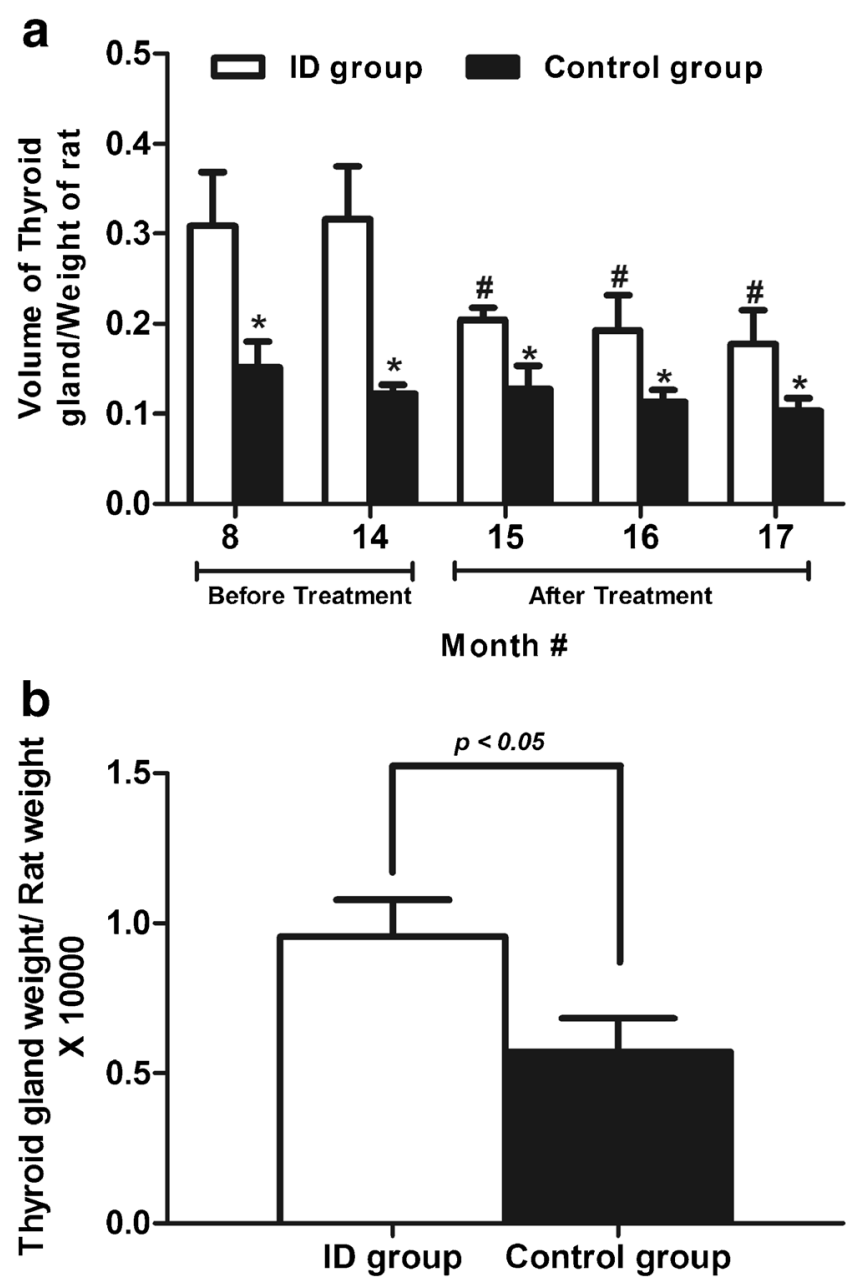

Fig. 6. a Ratio of volume of thyroid glands over weight of rats in iodine-deficient and control groups before treatment at months 8 and14 and after treatment at months 15, 16, and 17 (* indicates statistical significance between control group and ID group for each month and \# indicates statistical significance between ID group after treatment for months 15, 16 and 17 in comparison to month 14 before treatment). b Ratio of the thyroid gland weight over overall weight in iodine-deficient and control groups after sacrificing rats at the end of the study; all data are expressed as means $\pm \mathrm{SD}$

no change in $\mathrm{T}_{3}$ during the first few weeks and up to a month of the study (37). While $\mathrm{T}_{4}$ has shown decreasing values during iodine deficiency, $\mathrm{T}_{3}$ usually shows contradicting results $(36,38)$. Surprisingly in some studies, $\mathrm{T}_{3}$ decreases significantly within weeks or months while in other studies $T_{3}$ shows no changes in values (39). This was explained by the adaptive mechanism by which the thyroid gland and the extrathyroidal tissues preferentially secrete $T_{3}$ over $T_{4}$ or convert $T_{4}$ to $T_{3}$. Typically, $T_{4}$ has lower biological activity as compared to $\mathrm{T}_{3}$ (the active form of the thyroid hormones). It has been reported that $80 \%$ of $\mathrm{T}_{3}$ comes from $\mathrm{T}_{4}$ by the removal of one iodine atom from the outer benzene ring of $\mathrm{T}_{4}$ in the liver, kidneys, muscle, and central nervous system (40). After 4 months, the values of $\mathrm{T}_{3}$ and $\mathrm{T}_{4}$ in our study were $5.2 \pm 0.6$ and $233 \pm 9 \mathrm{ng} / \mathrm{mL}$ in the ID group and $7.9 \pm 1.6 \mathrm{ng}$ and $283 \pm 9$ in the control group. These values might not resemble reported hormone values in the literature, but that might become fathomable with a comprehensive review of the literature of thyroid hormones values that reveals high variation in published data $(4,36,37,39,41)$.
After 5 months of the study, $\mathrm{T}_{3}$ and $\mathrm{T}_{4}$ values continued to decline until reaching a minimum plateau at month 7 for $\mathrm{T}_{3}$ and at month 10 for $\mathrm{T}_{4}$ (Fig. 2a, b). This could be explained by the inability of the aforementioned intrathyroidal mechanisms (32) to compensate for the consistent depletion of iodide. Moreover, the constant concentration of $\mathrm{T}_{3}$ at a time when $\mathrm{T}_{4}$ values were still decreasing could be attributed to more $T_{4}$ is being converted to $T_{3}$ (the active form of thyroid hormones). This comes in agreement with a long term study by Riesco et al., where thyroid function was assessed up to 55 weeks intervals after the onset of iodine-deficient diet. In this study, serum $\mathrm{T}_{3}$ showed significant decrease to about $50 \%$ of the normal level after 7 weeks. However, no further decrease was appeared even at 55 weeks. While $\mathrm{T}_{4}$ showed a consistent decreasing pattern for periods longer than $\mathrm{T}_{3}(36)$.

$\mathrm{TSH}$, on the other hand, showed a typical response to what is expected during iodine deficiency cases. However, in our study, TSH during the first 2 months of the study did not show the expected behavior, as a significant reduction in thyroid hormones did not correlate to a significant increase in TSH values. It has been suggested that more dramatic drops in $\mathrm{T}_{4}$ over a longer duration may be necessary before observing a marked increase in TSH (41).

In a related study, low-iodide diet did not cause any significant change in $\mathrm{T}_{3}$ and $\mathrm{TSH}$ while $\mathrm{T}_{4}$ decreased by $50 \%$ (39). This could be attributed to the adaptive mechanisms mentioned earlier that allowed the thyroid system to compensate for the loss of thyroid hormones without triggering any TSH secretion. This was confirmed by Kouichi et al. study (40) that stated that intrathyroidal response mechanisms occur to compensate for the reduction of iodide without any significant increase in circulating TSH.

In our study, TSH levels showed a significant increase over the next few months. A plateau was reached at month 9 with a value of $7.8 \pm 2.6 \mathrm{ng} / \mathrm{mL}$ (Fig. 2c). This pattern agrees with the end points of $T_{3}$ and $T_{4}$ profiles at the pretreatment phase where both hormones reached a plateau at month 7 and month 10 of the study. After initiating the transdermal treatment of the iodide microemulsion in the ID animal group, and within the first month, the evidence for the influence of the treatment was clearly shown (Fig. 2). A sharp increase in the hormonal values of $\mathrm{T}_{3}$ and $\mathrm{T}_{4}$ was observed and this increase was associated with a sharp decrease in TSH values (Fig. 2). Unexpectedly, the increase in $\mathrm{T}_{3}$ and $\mathrm{T}_{4}$ levels surpassed that in the control group. This behavior can be associated with the high thirst of the thyroid enzymes to synthesize $T_{3}$ and $T_{4}$ as the exhausted tissues are trying to compensate for the months of depletion during the iodine deficiency phase. After the first month of treatment, the TSH value in the ID group matched the normal values of the control group. TSH tends to stimulate the thyroid gland to synthesize and secrete more hormones until the normal body requirements are met. By the third month of treatment, $\mathrm{T}_{3}$ and $\mathrm{T}_{4}$ values in the ID group further decreased to similar hormonal values of $T_{3}$ and $T_{4}$ in the control group. This might be attributed to a negative feedback mechanism that develops from the reduced levels of the thyroid stimulator TSH. A reduction in TSH values suppresses the thyroid gland from secreting any excess of $T_{3}$ and $T_{4}$. This indicates that the thyroid gland needed about 2 months to restore its normal hormonal functionality. This lag time for $\mathrm{T}_{3}$ and $\mathrm{T}_{4}$ values to reach normal levels comes in agreement 


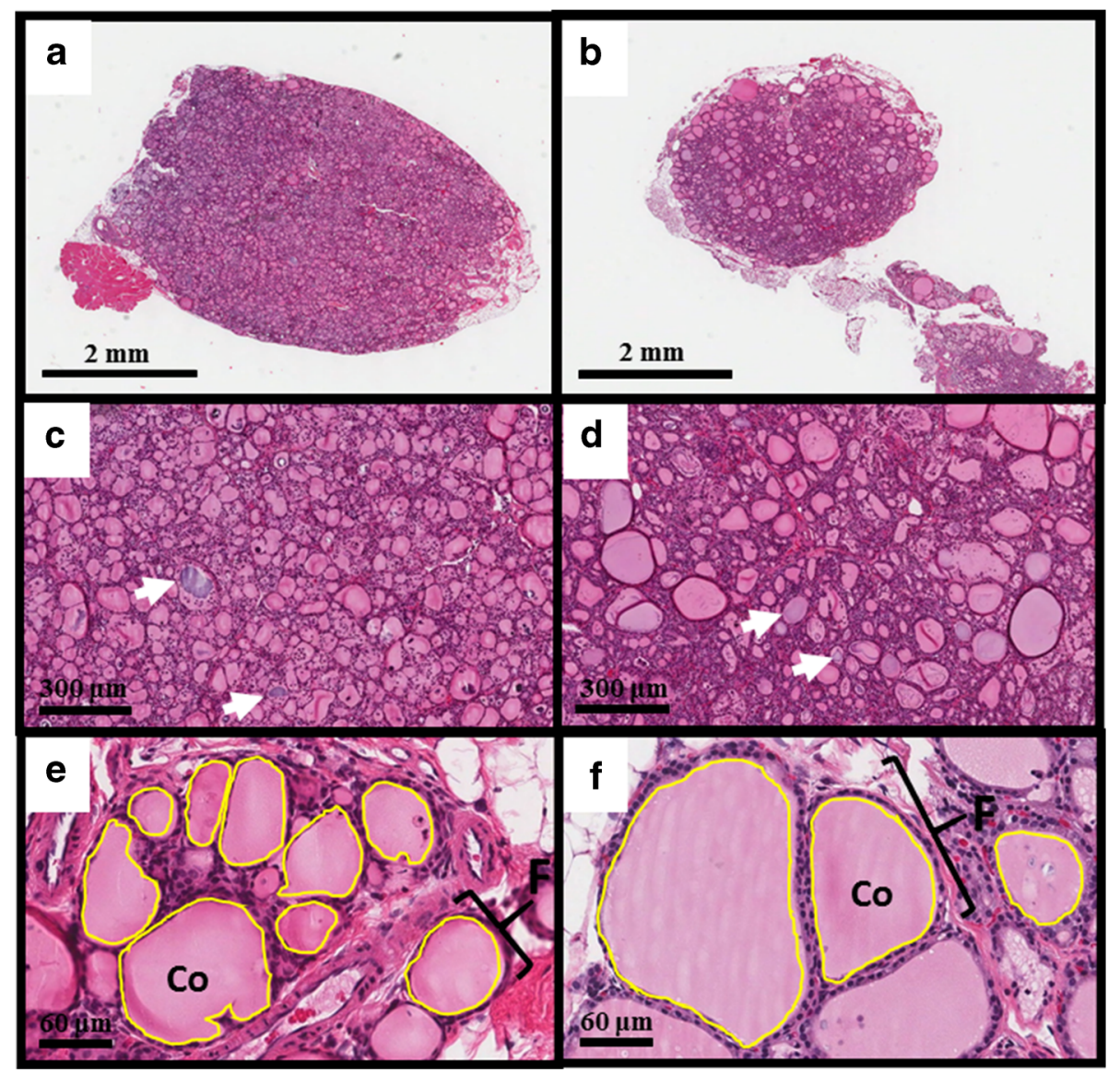

Fig. 7. Histology of thyroid gland. Coronal sections of a single thyroid lobe (hematoxylin and eosin) from a representative rat from the iodine-deficient group (a, c, e) and control group (b, d, f). Morphological size differences between the two groups are clear at low power (a, b at $2.0 \times$ magnification, scale is $2 \mathrm{~mm}$ ). At higher power (10.0×). Iodine-deficient group (c) shows a diffuse homogeneous pattern of follicular hyperplasia with limited volumes of stored colloid and control group (d) showing the variable-sized follicles and pools of colloid which is the expected pattern of a euthyroid gland (scale is $300 \mu \mathrm{m}$ ). The white arrows show examples of mineralization within the follicles present within both groups (c, d). Stereology example of histological sections (scale at $60 \mu \mathrm{m})(\mathbf{e}, \mathbf{f})$. Yellow outline shows colloid $(\mathrm{Co})$ area measured and thyroid follicles (f)

with a previous study where male Wistar rats were fed iodinedeficient diet for 6 months then they were replenished with

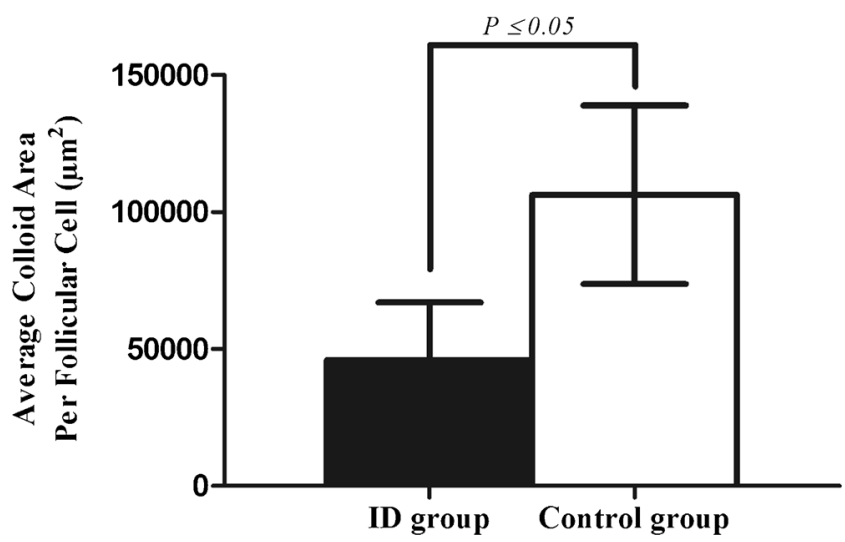

Fig. 8. The average colloid area $\left(\mu \mathrm{m}^{2}\right)$ per 30 individual thyroid follicles from each rat in ID and control groups. All image measurements were performed at $40.0 \times$ magnification. Final results are presented as group averages \pm standard deviations
$20-50 \mu \mathrm{g}$ of daily iodine for 140 days. It was stated that it took 2-3 months for the complete thyroid function to be restored. The same study also showed the TSH values reached normal levels after 1 month which is similar to our findings (42). It is important to note that the values of $\mathrm{T}_{4}$ and $\mathrm{TSH}$ in the control group remained consistent throughout the study. However, $\mathrm{T}_{3}$ values in the control group showed an unexpected increase during the treatment phase. This was surprising as we can speculate that this increase in $T_{3}$ values might be attributed to the aging effect of the rats or other metabolic disturbances in the tissues. Nevertheless, thyroid hormones $\mathrm{T}_{3}$ and $\mathrm{T}_{4}$ and TSH indicated a successful treatment of transdermal iodine delivery to restore normal functionality of the thyroid gland.

It is widely accepted that there is a strong correlation between iodine levels in human blood and iodide quantity excreted in urine. Iodide ions excreted in urine is the result of metabolic processing of thyroid hormones or due to an excess in plasma concentration after an increase in oral iodine uptake (43). It was reported that about $95 \%$ of the daily iodide intake in rats was excreted in urine (11). However, this percentage can change significantly in case of iodide 
deficiency. It was reported that during steady-state iodide deficiency (with diet of $1 \mu \mathrm{g} /$ day), the percentage of iodide excreted in urine as compared to daily iodide intake decreased to about $65 \%$. Nevertheless, measuring the iodide ion in a 24-h urine collections is still a standard method for the evaluation of iodine status (10). In this study and prior to the treatment phase, control and ID groups were fed diets that contain $200 \mu \mathrm{g}$ and $20 \mu \mathrm{g} / \mathrm{kg}$ of iodine. After the consumption of these two diets, significant differences in urinary iodine were observed between the two groups. In general, the ID group showed a significant reduction in the concentration of iodide in urine as compared to the control group (Fig. 3). Prior to the treatment phase, the average urinary iodide values in ID and control groups were $0.5 \pm 0.2$ and $3.6 \pm 2 \mu \mathrm{g} / \mathrm{mL}$, respectively. However, in the control group after month 6, iodide concentration started to decrease gradually until it reached a value of $1.5 \pm 0.1 \mu \mathrm{g} / \mathrm{mL}$ by the end of month 14 (Fig. 3). The differences between the two groups remained significant between months 4 and $14(P<0.05)$. Once the microemulsion treatment started on month 14 (ID group), a sharp increase in iodide concentration was reached with an average value of $8 \mu \mathrm{g} / \mathrm{mL}$ throughout the course of the treatment. Subsequently, this data provides second evidence that the iodide ion permeated through the skin and the microemulsion delivery system was bioavailable.

Since the main function of thyroid hormones is to control energy consumption of the body, body weight assessment is considered a good parameter for evaluating the response of the whole body to any changes in thyroid system. One of the reported symptoms of iodide deficiency in human is weight gain (44). However, it is totally different for rats. We noticed in our study that there were no significant differences in body weights between ID and control groups over the whole study period $(P \geq 0.05)$ (Fig. 4). Nevertheless, the growth rate for the ID group was lower as it took 7 months for the ID group to reach $500 \mathrm{~g}$, while the rats in the control group reached the same weight average in 3 months (Fig. 4). This comes in agreement with previous study that showed limited reduction in growth of the iodine-deficient rats during 7-18 weeks (45). Another study showed no significant changes between ID and control groups for a period of time of more than 1 year and the rats continued to gain weight at the same rate and remained healthy (38). These significant differences between humans and rats in weight gain/loss are not explained. More studies are needed to compute the total body fat between ID rats and the control. Transverse images of the thyroid gland at the widest level when scanned from caudal to cranial using ultrasound imaging showed clear evidence of hyperplasia exhibited in the ID group compared to that in the control group (Fig. 5a). Furthermore, mild enlargement of the gland is still evident following 13 weeks of treatment in the ID group (Fig. 5e). Structurally, the thyroid gland in the ID group shows a diffuse homogeneous pattern of follicular hyperplasia with limited volumes of stored colloid. In contrast, the variablesized follicles and pools of colloid from the control group is the expected pattern for a euthyroid rat. The progression of ultrasound changes post-treatment and the histology at the time of sacrifice reveal that, although the physiological parameters have returned to normal values, the thyroid requires greater than 13 weeks to return to a morphologically euthyroid state (Fig. 7b). The progressive shrinking of the thyroid volume following the initial 4 weeks of treatment was clear clinical evidence that the topical therapy was efficient. Further studies are needed to fully characterize the time course for anatomical measurements to fully normalize.

Mineralization is a normal aging change in these rats (513 days old at sacrifice). Special staining would be needed to better quantify the relative amounts of mineralization versus other possible basophilic material present within the follicles. Some of the fragmented material may be a result of the ID group hyperplastic glands normalizing through involution as previously described (46). The sloughing pyknotic cells were likely a result of post-mortem autolysis and could be avoided in the future with faster tissue isolation and immediate fixation in preservative, rather than taking the time to record weights and gross images as documented here. The mild parafollicular cell hyperplasia would require calcitonin staining to further classify the hyperplasia. However, due to the advanced age of the rats at time of tissue collection and the lack of pleomorphic nuclei, the parafollicular cell hyperplasia was most likely reactive, associated with age, rather than neoplastic. The bilateral parathyroid gland hyperplasia has been reported in aging rats (47) and is not of concern for this study.

From the stereology evaluation, the smaller standard deviation of the ID group is evidence of the homogeneity among follicular colloid compared to the expected more heterogeneous distribution of the euthyroid control follicles. To see the follicular colloid stores return to a normal distribution, additional experiments with monitoring after stopping treatment with microemulsion therapy would be needed. The follicular hyperplasia and reduced colloid stores in the ID group after 13 weekly treatments may support the possibility that the rats were overtreated with the microemulsion as discussed in earlier sections. Our initial intentions were to use a carefully projected animal dose that would be used later in a safety human study, to determine the duration that would bring the rats back to control hormonal values, and to show physiological and pathological signs of recovery. We expect a smaller dose will show similar effects in rats since the daily normal daily uptake of iodine in rats is about $2-3 \mu \mathrm{g}$ /day by oral route. We noticed from our previous in vitro study (Lou et al.) that only $10 \%$ of the total volume of microemulsion has penetrated through the human skin in $24 \mathrm{~h}$. We project that the skin will absorb the content of the microemulsion and will store it within the fatty cells beneath the skin. This behavior will be similar to an intramuscular injection.

\section{CONCLUSION}

Iodide deficiency is considered a global problem facing the health care system worldwide. In our study, here, we provided an easy and scalable approach to deliver iodide ion using topical microemulsion. The efficacy of microemulsion was evaluated by measuring the main physiological parameters related to iodide deficiency. This included thyroid hormones $\mathrm{T}_{3}, \mathrm{~T}_{4}$, and $\mathrm{TSH}$; urinary iodide concentration; body weight; and thyroid gland size. All these measurements confirmed the efficiency of microemulsion as a delivery system for transdermal delivery of iodide into and an effective approach to restoring the hormonal balance in the thyroid system. 
Although an animal model was utilized here, this approach can be easily transferable to humans.

\section{ACKNOWLEDGMENTS}

We would like to thank Stacey Barnett and Ernestine Hayes from Department of Comparative Medicine at UTHSC for helping with blood and urine collections. Special thanks are given to Dr. David Brand, Karen Whittington, and Amanda Prislovisky for giving us an access to the MAGPIX Luminex instrument from BioRad at the VA Hospital in Memphis. We would like to thank Dr. Tina M. Herfel from Harlan Laboratories for helping us with the selection of diets and Mr. Craig Lanio from EMD Millipore Corporation for assisting with thyroid hormones' raw data analysis. Special thanks are also given to Launch Your City Inc. in Memphis for the grant funding and the Center for Pediatric Pharmacokinetics and Therapeutics (CPPT) for providing the postdoctorate fellowship funding.

CONFLICT OF INTEREST The authors declare that they have no competing interests.

\section{REFERENCES}

1. Miot F, Dupuy C, Dumont JE, Rousset BA. Thyroid hormone synthesis and secretion. Thyroid Manag. 2010:1-38.

2. Anguiano B, García-Solís P, Delgado G, Velasco CA. Uptake and gene expression with antitumoral doses of iodine in thyroid and mammary gland: evidence that chronic administration has no harmful effects. Thyroid Off J Am Thyroid Assoc. 2007;17:851-9.

3. Spitzwegand C, Morris JC. Sodium iodide symporter (NIS) and thyroid. Hormones (Athens). 2002;1:22-34.

4. Hussein AE-AM, Abbas AM, El Wakil GA, Elsamanoudy AZ, El Aziz AA. Effect of chronic excess iodine intake on thyroid function and oxidative stress in hypothyroid rats. Can J Physiol Pharmacol. 2012;90:617-25.

5. Erkekoglu P, Giray BK, Caglayan A, Hincal F. Selenium and/or iodine deficiency alters hepatic xenobiotic metabolizing enzyme activities in rats. J Trace Elem Med Biol. 2012;26:36-41.

6. Zimmermann MB. Iodine requirements and the risks and benefits of correcting iodine deficiency in populations. J Trace Elem Med Biol. 2008;22:81-92.

7. Kleinand I, Ojamaa K. Thyroid hormone and the cardiovascular system. N Engl J Med. 2001;344:501-9.

8. Monaco F. Thyroid diseases. Taylor \& Francis; 2012.

9. Oppenheimer J. Molecular basis of thyroid hormone action. Elsevier; 2012.

10. Cicalese M, Bruzzese E, Guarino A, Spagnuolo M. Requesting iodine supplementation in children on parenteral nutrition. Clin Nutr. 2009;28:256-9.

11. McLanahan ED, Andersen ME, Fisher JW. A biologically based dose-response model for dietary iodide and the hypothalamicpituitary-thyroid axis in the adult rat: evaluation of iodide deficiency. Toxicol Sci. 2008;102:241-53.

12. Dietrich JW, Landgrafe G, Fotiadou EH. TSH and thyrotropic agonists: key actors in thyroid homeostasis. J Thyroid Res. 2012.

13. Lumen A, Mattie DR, Fisher JW. Evaluation of perturbations in serum thyroid hormones during human pregnancy due to dietary iodide and perchlorate exposure using a biologically based doseresponse model. Toxicol Sci. 2013;133:320-41.

14. Untoro J, Timmer A, Schultink W. The challenges of iodine supplementation: a public health programme perspective. Best Pract Res Clin Endocrinol Metab. 2010;24:89-99.
15. Maier J, Van Steeg H, Van Oostrom C, Paschke R, Weiss R, Krohn K. Iodine deficiency activates antioxidant genes and causes DNA damage in the thyroid gland of rats and mice. Biochim Biophys Acta (BBA) Mol Cell Res. 2007;1773:9909.

16. Morand S, Chaaraoui M, Kaniewski J, Dème D, Ohayon RE, Noel-Hudson MS, et al. Effect of iodide on nicotinamide adenine dinucleotide phosphate oxidase activity and Duox 2 protein expression in isolated porcine thyroid follicles. Endocrinology. 2003;144:1241-8.

17. Clero E, Doyon F, Chungue V, Rachedi F, Boissin JL, Sebbag J, et al. Dietary iodine and thyroid cancer risk in French Polynesia: a case-control study. Thyroid Off J Am Thyroid Assoc. 2012;22:422-9.

18. U. WHO. ICCIDD. Assessment of the iodine deficiency disorders and monitoring their elimination. World Health Organization, Geneva, Switzerland WHO/NHD/011(2001); 2001.

19. Zimmermannand MB, Andersson M. Assessment of iodine nutrition in populations: past, present, and future. Nutr Rev. 2012;70:553-70.

20. Zimmermann MB. Iodine deficiency. Endocr Rev. 2009;30:376408.

21. Zimmermann MB, Andersson M. Update on iodine status worldwide. Curr Opin Endocrinol Diabetes Obes. 2012;19:382-7. 310.1097/MED.1090b1013e328357271a.

22. Abraham GE, Tlechas J, Hakala J. Measurement of urinary iodide levels by ion-selective electrode: improved sensitivity and specificity by chromatography on an ion-exchange resin. Original Internist. 2004;11:19-32.

23. Banksand MR, Farthing MJ. Fluid and electrolyte transport in the small intestine. Curr Opin Gastroenterol. 2002;18:176-81.

24. Dasgupta PK, Liu Y, Dyke JV. Iodine nutrition: iodine content of iodized salt in the United States. Environ Sci Technol. 2008;42:1315-23.

25. Zimmermann MB. Iodine: it's important in patients that require parenteral nutrition. Gastroenterology. 2009;137:S36-46.

26. McNeill SC, Potts RO, Francoeur ML. Local enhanced topical delivery (LETD) of drugs: does it truly exist? Pharm Res. 1992;9:1422-7.

27. Lou H, Qiu N, Crill C, Helms R, Almoazen H. Development of W/O microemulsion for transdermal delivery of iodide ions. AAPS PharmSciTech. 2013;14:168-76.

28. Narayanan L, Buttler GW, Yu KO, Mattie DR, Fisher JW. Sensitive high-performance liquid chromatography method for the determination of low levels of perchlorate in biological samples. J Chromatogr B Anal Technol Biomed Life Sci. 2003;788:393-9.

29. Mancini M, Vergara E, Salvatore G, Greco A, Troncone G, Affuso A, et al. Morphological ultrasound microimaging of thyroid in living mice. Endocrinology. 2009;150:4810-5.

30. Kot BCW, Lau TYH, Cheng SCH. Stereology of the thyroid gland in Indo-Pacific bottlenose dolphin (Tursiops aduncus) in comparison with human (Homo sapiens): quantitative and functional implications. PLoS One. 2013;8:e62060.

31. Morell SF. The great iodine debate. Wise traditions in food, farming, and the healing arts, summer. 2009.

32. Wolffand J, Chaikoff I. Plasma inorganic iodide as a homeostatic regulator of thyroid function. J Biol Chem. 1948;174:555-64.

33. Abraham GE. The bioavailability of iodine applied to the skin. Internist. 2002;9:30-41.

34. Gentile F, Di Lauro R, Salvatore G. Biosynthesis and secretion of thyroid hormones. Endocrinology. 1995;1:517.

35. Kunisue T, Fisher JW, Kannan K. Modulation of thyroid hormone concentrations in serum of rats coadministered with perchlorate and iodide-deficient diet. Arch Environ Contam Toxicol. 2011;61:151-8.

36. Riesco G, Taurog A, Larsen PR, Krulich L. Acute and chronic responses to iodine deficiency in rats 1. Endocrinology. 1977;100:303-13.

37. Fukuda H, Yasuda N, Greer M, Kutas M, Greer S. Changes in plasma thyroxine, triiodothyronine, and TSH during adaptation to iodine deficiency in the rat. Endocrinology. 1975;97:307-14. 
38. Greer MA, Grimm Y, Studer H. Qualitative changes in the secretion of thyroid hormones induced by iodine deficiency. Endocrinology. 1968;83:1193-8.

39. Abramsand G, Larsen P. Triiodothyronine and thyroxine in the serum and thyroid glands of iodine-deficient rats. J Clin Investig. 1973;52:2522.

40. Minato K, Nagao H, Imazu T, Takahashi K. Influence of iodinedeficiency on thyroid hormones homeostasis in rats. Biol Pharm Bull. 2012;35:1166-70.

41. Gilbert M, McLanahan E, Hedge J, Crofton K, Fisher J, ValentínBlasini L, et al. Marginal iodide deficiency and thyroid function: dose-response analysis for quantitative pharmacokinetic modeling. Toxicology. 2011;283:41-8.

42. Rognoni J, Lemarchand-Béraud T, Berthier C, Simon C. Effect of long-term iodide refeeding on the synthesis and secretion of T3, T4 and TSH in severe iodine deficient rats. Acta Endocrinol (Copenh). 1982;101:377-85.
43. McLanahan ED, Campbell JL, Ferguson DC, Harmon B, Hedge JM, Crofton KM, et al. Low-dose effects of ammonium perchlorate on the hypothalamic-pituitary-thyroid axis of adult male rats pretreated with PCB126. Toxicol Sci. 2007;97:308-17.

44. Feligand P, Frohman LA. Endemic goiter. Endocrinology \& metabolism McGraw-Hill Professional; 2001.

45. McIntosh G, Jones G, Howard D, Belling G, Potter B, Hetzel B. Low-iodine diet for producing iodine deficiency in rats. Aust $\mathrm{J}$ Biol Sci. 1980;33:205-12.

46. Tachiwakiand O, Wollman SH. Shedding of dense cell fragments into the follicular lumen early in involution of the hyperplastic thyroid gland. Lab Invest $\mathrm{J}$ Techn Methods Pathol. 1982;47:91-8.

47. Halloran B, Udén P, Duh Q-Y, Kikuchi S, Wieder T, Cao J, et al. Parathyroid gland volume increases with postmaturational aging in the rat. Am J Physiol Endocrinol Metab. 2002;282:E557-63. 